\title{
Response to Drs. Ohayon and Guilleminault
}

Naresh M. Punjabi, MD, PhD; Brock A. Beamer, MD

Johns Hopkins University, Baltimore, MD

\section{DEAR EDITOR,}

WE THANK DRS. OHAYON AND GUILLEMINAULT FOR THEIR COMMENTS ON OUR ARTICLE ON THE ASSOCIATION BETWEEN SLEEP-DISORDERED BREATHING (SDB) and $\mathrm{C}$-reactive protein (CRP). ${ }^{1}$ We share their concern that to discern the role of SDB in systemic inflammation, the effects of adiposity should be carefully considered. In fact, numerous studies have shown that both total body fat and the amount of visceral fat are strong correlates of CRP levels. ${ }^{2-5}$ Thus, inclusion of obesityrelated indices in the analysis of observational data relating SDB to CRP levels is absolutely essential. The scientific challenge is to determine which aspects of obesity should be accounted for in such analyses. Inclusion of several variables, especially correlated variables, in a statistical model can lead to overadjustment and multi-collinearity. Conversely, exclusion of variables that are biologically important can lead to concerns of residual confounding from factors that are omitted or inadequately controlled. Therefore, there is an obvious tension between the need to construct the most parsimonious statistical model and the need to adjust for scientifically relevant variables. Recognizing the influence of obesity on CRP levels, we opted to adjust for obesity using various measures simultaneously. Parameter estimates resulting from such models with collinear variables will have inflated standard errors. Large standard errors make it difficult to identify "statistically significant" associations and often require acceptance of null hypotheses. By including the apnea-hypopnea index (AHI), BMI, waist circumference, and percent of body fat in our models, we biased against finding an independent association between SDB severity and CRP levels. Furthermore, the decision to include the different indices of obesity was driven by the concern for the effects of body fat amount and distribution on CRP levels. To decrease multi-collinearity, we have repeated our analyses by including each of the obesity measures individually and in all possible pairwise combinations with AHI as predictors of CRP levels. Irrespective of the set of obesity variables used, our conclusion of an independent association between SDB severity and CRP levels remains materially unchanged.

It is also argued that the BMI of the sample was high and hence

\section{Disclosure Statement}

Drs. Punjabi and Beamer have indicated no conflicts of interest.

\section{Submitted for publication March 2007 \\ Accepted for publication March 2007}

Address correspondence to: Naresh M. Punjabi, MD, PhD, Department of Medicine, Johns Hopkins University, 5501 Hopkins Bayview Circle, Baltimore, MD 21224; Tel: (410) 550-5405; Fax: (410) 550-2612; E-mail: npunjabi@jhmi.edu adjustment for BMI does not circumvent the concern for confounding due to obesity. Indeed, the median BMI of the sample was $30.5 \mathrm{~kg} / \mathrm{m}^{2}$ (interquartile range: $27.2-35.0 \mathrm{~kg} / \mathrm{m}^{2}$ ). Although the sample was predominantly overweight or obese, this issue by itself does not invalidate our conclusions on SDB and CRP levels. Surely, the lack of variation in one predictor (i.e., BMI) does not completely dissolve the possibility that there is some level of unique variation in CRP levels that can be accounted for by another predictor (i.e., AHI). The fact that the sample was overweight and obese merely limits the generalizability and suggests that in this subgroup, CRP levels vary as a function of the AHI.

Another concern raised is that there are other factors that were not adequately considered such as habitual sleep duration and cigarette smoking. Here again, we agree that there is an enlarging list of factors that have causal relevance with regards to increasing CRP levels. It was this very concern that led us to exclude a majority of health conditions that are prevalent in patients with SDB. The strict exclusion criteria greatly limited our capability to identify a large enough sample across the spectrum of SDB severity. Imposing additional constraints and/or assessing other measures (e.g., habitual sleep duration) are not insurmountable tasks. The enlarging list of variables that can influence CRP levels is actually welcomed by many as it provides a roadmap for future research for those entrenched in understanding the complex pathophysiology of SDB-related adverse outcomes.

So did our study shed absolutely no new light on the topic of SDB and systemic inflammation? On the contrary, this collegial debate is telling evidence that our study has had an impact as it has highlighted the paucity of observational and experimental data on CRP levels in SDB. We admit that we are in equipoise on whether SDB is a causal correlate of higher CRP levels. It seems to us that by demonstrating an independent SDB-CRP association, albeit in a non-generalizable sample, we are making headway toward the kind of clarity required in future research efforts on the causal effects of SDB in systemic inflammation. Additional research is obviously necessary and it is a tricky task. Ohayon and Guilleminault remind us to pay more attention to the fundamental issues of observational research and indirectly highlight many ways that future studies can be better designed and analyzed. We thank our esteemed colleagues for their thoughtful comments and welcome continued discussion on these important topics.

\section{REFERENCES}

1. Punjabi NM, Beamer BA. C-reactive protein is associated with sleep disordered breathing independent of adiposity. Sleep 2007;30:29-34 .

2. Festa A, D'Agostino R Jr, Williams K, et al. The relation of body fat mass and distribution to markers of chronic inflammation. Int $\mathrm{J}$ Obes Relat Metab Disord 2001;25:1407-15.

3. Rexrode KM, Pradhan A, Manson JE, Buring JE, Ridker PM. Relationship of total and abdominal adiposity with CRP and IL-6 in women. Ann Epidemiol 2003;13:674-82. 
4. Saijo Y, Kiyota N, Kawasaki Y, et al. Relationship between C-reactive protein and visceral adipose tissue in healthy Japanese subjects. Diabetes Obes Metab 2004;6:249-58.

5. Panagiotakos DB, Pitsavos C, Yannakoulia M, Chrysohoou C, Stefanadis C. The implication of obesity and central fat on markers of chronic inflammation: The ATTICA study. Atherosclerosis 2005;183:308-15. 